\title{
Aufsätze
}

\section{Hubert Treiber}

\section{Verwaltungsrechtswissenschaft}

als Steuerungswissenschaft -

eine »Revolution auf dem Papier«?

Anmerkungen zu einem intendierten Paradigmawechsel und zur »Kühnheit« von Schlüsselbegriffen ${ }^{1}$ (Teil 1)

Der Beitrag setzt sich mit der intendierten Reform des Verwaltungsrechts und der damit verbundenen Konzeptualisierung der Verwaltungsrechtswissenschaft als Stenerungswissenschaft kritisch auseinander. Zunächst wird erörtert, inwieweit es bei der für die Rechtswissenschaften typischen Trennung zwischen Theorie und Praxis einer "Umweghandlung" bedarf, damit von Wissenschaftlern gemachte "Inventionen "von der Praxis akzeptiert und damit zu »Innovationen "werden. Damit gerät die Formierung jener Trägerschicht in den Blickpunkt, die mit der Rezeption eines sozialwissenschaftlich eingefärbten Steuerungskonzepts, welche zu ibrer erkenntnistheoretischen Fundierung sog. Schlüsselbegriffe heranzieht, die Neuausrichtung von Verwaltungsrecht und Verwaltungsrechtswissenschaft in die Wege leitete. Damit verbunden ist die eher grundsätzliche Frage, welche Formen disziplinübergreifender Kommunikation eine relativ geschlossene Disziplin wie die Rechtswissenschaften überhaupt zulässt, zugleich stellt sich die eher spezifische Frage, wie es mit der erkenntnistheoretischen Fundierung der "Schlüsselbegriffe", zu denen auch der Steuerungsbegriff zählt, bestellt ist. Die ibnen zugeschriebene Funktion einer Transferleistung von einem Wissenschaftsfeld in ein anderes lenkt den Blick auf gängige moderne kognitive Metapherntheorien, die vor allem theoriekonstitutiven Metaphern im Entdeckungszusammenhang eine erkenntnisfördernde, heuristische Funktion zuweisen. Da im Reformdiskurs die explizit vorgenommene Bezugnabme zu modernen Metapherntheorien nicht zu erkennen ist, liegt es nabe, der reflektierenden Urteilskraft die Fülle der "Forschungsheuristiken " anzulasten, die neben durchaus trivialen Einsichten dem Schlüsselbegriff der Steuerung nicht abzusprechen sind, zumal auch eine aus der Sicht der Herkunftsdisziplin unzulängliche Rezeption durchaus kreativ sein kann. Wenn nunmebr der Stenerungsbegriff als "normativer Zurechnungs- und Rechtsfolgenzusammenhang "verstanden wird, ist außerdem zu erörtern, welche Konsequenzen dies hat.

I Lutz Danneberg (Berlin), Thomas Groß (Gießen), Günther Schmid (Berlin), Christoph Schönberger (Konstanz), Kay Waechter (Hannover) und Gerhard Wagner (Frankfurt) haben eine erste Fassung kritisch begutachtet. Christoph Schönberger und Kay Waechter standen darüber hinaus als geduldige Diskussionspartner zur Verfügung. Ihnen allen sei hierfür gedankt. Die Verantwortung für Mängel und Schwächen liegt jedoch allein bei mir. 
Im Vorwort zu dem von G.F. Schuppert herausgegebenen Sammelband mit dem Titel »Jenseits von Privatisierung und >schlankem $<$ Staat $\aleph^{2}$ aus dem Jahre 1999 findet sich eine bemerkenswerte Textpassage, die dem Leser nicht vorenthalten werden soll:

»Es gibt Begriffe, die - manchmal sogar zum eigenen Erstaunen ihrer Erfinder und Förderer - Karriere machen, Begriffe also, die entweder ihre Karriere schon hinter sich haben oder sich anschicken, sich ganz oben in der Erfolgsliste gelungener Begriffsbildungen zu etablieren. (...). - (...) man (beginnt) darüber nachzudenken, was eigentlich ein Begriff für Eigenschaften mitbringen muß, um Erfolg zu haben; (...).«3

$\mathrm{Zu}$ einem solchen »Erfolgsbegriff « zählt auch der Begriff der »Steuerung «, über dessen Verwendung und Karriere, aber auch Funktion im Verlauf der in den I980er Jahren einsetzenden Diskussion zur Reform des Verwaltungsrechts in diesem Beitrag nachgedacht werden soll. So soll danach gefragt werden, welche »Eigenschaften« ein solcher Begriff »mitbringen muss, um Erfolg zu haben«, doch sollen auch jene Voraussetzungen thematisiert werden, die jenseits »begrifflicher Eigenschaften« eine gewichtige Rolle spielen, wenn nach einer Erklärung gesucht wird, warum gerade dieser Begriff so erfolgreich gewesen ist, d. h. der verwaltungsrechtlichen Reformdebatte selbst zu ihrem bisherigen Erfolg verholfen hat, wobei freilich noch zu fragen wäre, was unter »Erfolg « hier genau zu verstehen ist (Invention oder Innovation).

Der Versuch, sich mit den »Eigenschaften« dieses Erfolgsbegriffs näher zu beschäftigen, weist insgesamt in eine Richtung, die fürs erste dadurch angedeutet werden soll, dass auf die von den beiden Herausgebern der 1998 erschienenen Aufsatzsammlung »Physiologie und industrielle Gesellschaft «, 4 P. Sarasin und J. Tanner, verfasste Einleitung verwiesen wird. Es handelt sich dabei um eine Textstelle, bei der die beiden Herausgeber kurz auf den in dem Sammelband abgedruckten Beitrag »Zur Physiologie des Rechts oder der Muskel als Scharnierbegriff «s eingehen und hierzu folgende Ausführungen machen:

\begin{abstract}
»Aber auch in anderen Disziplinen, wie zum Beispiel der Rechtswissenschaft (...), kommt es zum Kontakt mit dem physiologischen Diskurs. Dabei ist interessant, wie dies geschah. Hubert Treiber zeigt, daß physiologische Begriffe und Denkmodelle in einer Art und Weise übernommen bzw. saufgegriffen w werden, die man nicht als eine präzise und rational berechnete bezeichnen kann, sondern im Gegenteil als assoziative. (...). Der Hauptertrag der Studie liegt (...) in der Untersuchung der Modalitäten des Wissenstransfers von einer Wissenschaft zur anderen. Diese assoziativen >Übertragungswege< zeigen, wie ein wissenschaftlicher Diskurs von Metaphern strukturiert wird, von diskursiven >Plots`, die nützlich und evident erscheinen, die er aber in ihrer assoziativen, das heißt Bezüge und Konnotationen ermöglichenden und damit weitere Ideen generierenden Potenz (nicht) überblickt (...). Jedenfalls kann das assoziative Potential von übernommenen Metaphern zwar, wie im Falle der Jurisprudenz, in einer spekulativen Sackgasse enden, es kann aber auch, wie Treiber ebenfalls zeigt, über diesen Umweg vollständig neue Formen des Denkens provozieren: gemeint ist Freuds Topik des Unbewußten und der Trieborganisation, die nach dem Modell einer thermodynamischen Energiemaschine gedacht wird. « ${ }^{6}$
\end{abstract}

Von J. Gessinger stammt der Hinweis, dass »Metaphern (...) nicht ohne Grund das bevorzugte Mittel in Paradigmenschlachten (sind).«7 Wo solche Schlachten

\footnotetext{
2 Mit dem Untertitel »Verantwortungsteilung als Schlüsselbegriff eines sich verändernden Verhältnisses von öffentlichem und privatem Sektor.«

3 Schuppert (Fn. 2), S. 7 .

4 Sarasin/Tanner (Hrsg.), Physiologie und industrielle Gesellschaft. Studien zur Verwissenschaftlichung des Körpers im 19. und 20. Jahrhundert, I998.

5 Sarasin/Tanner (Fn. 4), S. I $70 \mathrm{ff}$.

6 Sarasin/Tanner (Fn. 4), S. 3 I f., Einleitung.

7 Gessinger, Metaphern in der Wissenschaftssprache, in: T. Bungarten (Hrsg.), Beiträge zur Fachsprachenforschung. Sprache in Wissenschaft und Technik, Wirtschaft und Rechtswesen, 1992, S. 47.
} 
um Paradigmenwechsel ausgetragen werden, bedarf es einer sich formierenden Trägerschicht, welche das neue Paradigma propagiert und durchzusetzen versucht, wobei (theoriekonstitutive oder propagandistische) Metaphern Formierung und Zusammenhalt einer solchen Trägerschicht erleichtern helfen. Da die fragliche Trägerschicht der Reformjuristen innerhalb der juristischen Disziplin agiert, ist einerseits danach $\mathrm{zu}$ fragen, welche Voraussetzungen gegeben sein müssen, damit aus einer schöpferischen Invention tatsächlich eine Innovation wird und die Invention nicht als »Revolution auf dem Papier« (Cohen) endet. Andererseits sind ebenso Überlegungen berechtigt, die sich der Frage zuwenden, welche disziplinüberschreitenden Austauschformen eine normative und relativ geschlossene Disziplin wie die Rechtswissenschaften, wenn überhaupt, typischerweise zulässt, wenn - wie im vorliegenden Fall - der Steuerungsbegriff (das Steuerungsmodell) von einer anderen Disziplin (genauer: von einer bestimmten Richtung innerhalb der Sozial- resp. Politikwissenschaft) übernommen wird. Da jeder Paradigmawechsel gegen eine bislang vorherrschende Position gerichtet ist, sollte diese auch benannt werden. Dank der von A. Voßkuhle vorgelegten Beiträge ${ }^{8}$ und der von $\mathrm{H}$. Schulze-Fielitz besorgten Besprechung des »Handbuchs des Staatsrechts « lassen sich jedoch Anhaltspunkte dafür gewinnen, gegen welche Position sich die sozialwissenschaftlich eingefärbte Reform des Verwaltungsrechts richtet. 9

Dem deutschen Verwaltungsrecht ist in den letzten Jahrzehnten immer wieder

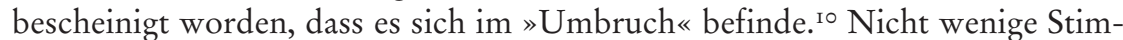
men, darunter diejenigen reputierlicher Fachvertreter, führen die notwendig erachtete Reform des Verwaltungsrechts u. a. auf eine »Krise« des sog. »Ordnungsrechts « zurück. In diesem Zusammenhang ist auch die Rede von einer Krise des »regulativen « Rechts, worunter »jene Gruppe von Normen« fällt, die »mit Geboten, Genehmigungsvorbehalten oder Strafandrohungen eine gezielte Wirkung bei den jeweiligen (Norm-)Adressaten auslösen wollen (...). «I ${ }^{\mathrm{I}}$ Die dem klassischen Ordnungsrecht bescheinigte Krise wurde nicht zuletzt durch die Ergebnisse einer Reihe von Implementationsstudien aus den I980er Jahren ins Bewusstsein gerufen, die vor allem dem damals neu geschaffenen Umweltrecht Vollzugsdefizite bescheinigten und zur Entdeckung informalen Verwal-

8 Vgl. Voßkuhle, `Schlüsselbegriffe $<$ der Verwaltungsrechtsreform. Eine kritische Bestandsaufnahme, Verwaltungsarchiv 92 (200I), S. I 86 ff.; Voßkuhle, \ I Neue Verwaltungsrechtswissenschaft, in: HoffmannRiem/Schmidt-Aßmann/Voßkuhle (Hrsg.), Grundlagen des Verwaltungsrechts. Bd. I, Methoden - Maßstäbe - Aufgaben - Organisation. München 2006, S. 8 ff. (Grundlagen).

9 Schulze-Fielitz, Grundsatzkontroversen in der Deutschen Staatsrechtslehre nach 50 Jahren Grundgesetz - in der Beleuchtung des Handbuchs des Staatsrechts, Die Verwaltung (künftig DV) 32 (I999), S. 24I ff.

Io Vgl. Bauer, Verwaltungsrechtslehre im Umbruch? Rechtsformen und Rechtsverhältnisse als Elemente einer zeitgemäßen Verwaltungsrechtsdogmatik, DV 25 (I992), S. 30I ff.; weitere Stimmen (Auswahl): Henke, Wandel der Dogmatik des öffentlichen Rechts, JZ 47 (I992), S. 54I ff.; Hoffmann-Riem, Reform des Allgemeinen Verwaltungsrechts: Vorüberlegungen, DVBl. го9 (I994), S. I 38 I ff.; Möllers, Braucht das Öffentliche Recht einen neuen Methoden- und Richtungsstreit?, Verwaltungsarchiv 90 (I999), S. I 87 ff.; Thieme, Über die Notwendigkeit einer Reform des Allgemeinen Verwaltungsrechts, DÖV 49 (I996), S. 757 ff.; Vesting, Reform des Allgemeinen Verwaltungsrechts. Bericht über die Konferenz in Hamburg am 22. u. 23. Nov. I99I, DVBl. I07 (I992), S. I 34 ff. Siehe bereits: Bachof, Die Dogmatik des Verwaltungsrechts vor den Gegenwartsaufgaben der Verwaltung, VVDStRL 30 (I972), S. I93 ff., sowie Brohm, Die Dogmatik des Verwaltungsrechts vor den Gegenwartsaufgaben der Verwaltung, VVDStRL 30 (1972), S. $245 \mathrm{ff}$.

I I Hierzu und weiter unten: Voßkuhle 200I, (Fn. 8), S. I 86. 

schaft gerade mit dieser Entdeckung anfänglich besonders schwer, so vermag in der Rückschau eine distanzierte Betrachtungsweise die Karriere dieses Begriffs bis zu seiner »Einhegung « durch das Recht unaufgeregt nachzuzeichnen. ${ }^{13}$ Voßkuhle ist zuzustimmen, wenn er feststellt, dass Reformen im allgemeinen »ihre Legitimation regelmäßig aus tatsächlichen oder vermeintlichen >Krisen« beziehen. So dürfte es kein Zufall sein, dass sich auch jene Reform des Allgemeinen Verwaltungsrechts, welche die »Verwaltungrechtswissenschaft (zur) Steuerungswissenschaft " umrüsten will, ${ }^{14}$ auf die Krise der herkömmlichen Ordnungsverwaltung beruft und hierbei in erster Linie auf die zum Umweltrecht durchgeführten Implementationsstudien verweist. ${ }^{\text {Is }}$ Diese Studien und ihr Untersuchungsgegenstand sind vor allem deshalb einschlägig, weil auf dem »Felde (des Umweltrechts) und an dessen Beispiel die Steuerungsfrage vor allem in das öffentliche Recht hineingetragen worden ist. «16 Ähnlich äußert sich Hoffmann-Riem, der deshalb ausführlich zu Wort kommen soll, weil er darüber hinaus die mit der Steuerungsfrage intendierte Perspektivenerweiterung und damit eines der wichtigsten Grundanliegen der vorgesehenen Reform des Verwaltungsrechts anspricht, so dass gerade diese Textstelle zugleich eine Anleitung dafür gibt, wie die später zu behandelnde Übertragung des einfachen Steuerungsmodells, wie von Renate Mayntz in einer Veröffentlichung aus dem Jahre I 987 vorgegeben, ${ }^{17} \mathrm{zu}$ lesen ist:

»Die von Sozialwissenschaftlern geprägte Frage nach dem Normvollzug setzt viel umfassender an als die traditionelle Frage der Juristen nach der Rechtmäßigkeit des Verhaltens der Umweltbehörden oder die rechtssoziologische Frage nach der Effektivität des Rechts. Die aus dem angelsächsischen Raum inspirierten - Implementationsforscher waren an politischer Steuerung (!) interessiert; sie erweiterten den Blick vom Gesetz auf das >Programm< unter Einschluß politischer Absichtserklärungen, administrativer Vorverständnisse und Verwaltungsübungen; sie lösten sich von der Fixierung auf den Subsumtionsakt und betrachteten die verschiedenen Stadien des gesamten Entscheidungsprozesses und deren Wechselbezüglichkeit; sie beobachteten Folgen und Folgesfolgen der Entscheidungen und insbesondere dysfunktionale Nebenfolgen; sie interessierten sich für das Wechselspiel von Programmverwirklichung und Programmentstehung und verabschiedeten mechanistische Analysekonzepte (!);

I2 Statt vieler: Mayntz/Derlien/Bohne/Hesse/Hucke/Müller, Vollzugsprobleme der Umweltpolitik. Empirische Untersuchung der Implementation von Gesetzen im Bereich der Luftreinhaltung und des Gewässerschutzes, I978; folgenreich waren vor allem die beiden Veröffentlichungen von Bohne, Informales Verwaltungshandeln im Gesetzesvollzug, in: Blankenburg/Lenk (Hrsg.), Organisation und Recht. Organisatorische Bedingungen des Gesetzesvollzugs. Jahrbuch für Rechtssoziologie und Rechtstheorie, Bd. 7 , I980, S. 20 ff., sowie Bohne, Der informale Rechtsstaat. Eine empirische und rechtliche Untersuchung zum Gesetzesvollzug unter besonderer Berücksichtigung des Immissionsschutzes, I98 I. Für die Rezeption durch die Rechtswissenschaften höchst einschlägig war der Vortrag Hoffmann-Riems vor der Staatsrechtslehrervereinigung, vgl. Hoffmann-Riem, Selbstbindungen der Verwaltung, VVDStRL $4 \circ$ (1982), S. I $87 \mathrm{ff}$.

I 3 Siehe Dreier, Informales Verwaltungshandeln, Staatswissenschaften und Staatspraxis 4 (I993), S. 647 ff.

I4 Schuppert, Verwaltungsrechtswissenschaft als Steuerungswissenschaft, in: Hoffmann-Riem/SchmidAßmann (Hrsg.), Reform des Allgemeinen Verwaltungsrechts. Grundfragen, I993, S. 65 ff. Ferner: Schuppert, Recht als Steuerungsinstrument: Grenzen und Alternativen rechtlicher Steuerung, in: Ellwein/Hesse (Hrsg.), Staatswissenschaften. Vergessene Disziplin oder neue Herausforderung, I990, S. $73 \mathrm{ff}$.

Is Diese Krise lässt sich auch als »Steuerungskrise« darstellen. Vgl. Klement, Verantwortung. Funktion und Legitimation eines Begriffs im Öffentlichen Recht, 2006, S. I 3 ff.

I6 Schmidt-Aßmann, Zur Funktion des Allgemeinen Verwaltungsrechts. Ein Exposé, DV 27 (1994), S. $137 \mathrm{ff}$.

I7 Bei der Abhandlung von Mayntz, Politische Steuerung und gesellschaftliche Steuerungsprobleme - Anmerkungen zu einem theoretischen Paradigma, Jahrbuch zur Staats- und Verwaltungswissenschaft, Bd. I (1987), S. 89 ff., handelt es sich um einen Basistext, auf den sich die Reformjuristen lange Zeit bezogen. Scharpf (Politiknetzwerke als Steuerungssubjekte, in: Derlien/Gerhardt/Scharpf (Hrsg.), Systemrationalität und Partialinteresse. Festschrift für Renate Mayntz, I994, S. 38 I) verwendet den Ausdruck Steuerungsmodell für den von R. Mayntz unterbreiteten Vorschlag, wir folgen ihm darin. 
sie untersuchten Handlungsstrategien und -situationen sowie die organisatorischen und verfahrensmäßigen Rahmenbedingungen des Rechts. «18

An dieser Stelle sei nur soviel vorweggenommen. Es ist offensichtlich, dass es hierbei um eine grundsätzliche Neuausrichtung des Verwaltungsrechts geht, die auf ein komplexes Wirkungsgefüge samt gegebenen Wechselwirkungen ausgerichtet ist, das nicht nur die jeweils agierenden (staatlichen und nicht-staatlichen) Akteure mit einschließt, sondern beispielsweise auch das Verwaltungsorganisationsrecht, das Verwaltungsverfahren sowie die zur Verfügung stehenden Finanzmittel unter Steuerungsgesichtspunkten umfassend einbezieht. Insofern ist ein Paradigmawechsel angesagt, dessen wichtigstes Anliegen allerdings schon viel früher artikuliert wurde. ${ }^{19}$

Anders als Voßkuhle, welcher als wichtige Antriebskräfte für die intendierte Verwaltungsrechtsreform das »innenpolitische Reformklima«, die »Europäisierung des nationalen Verwaltungsrechts « sowie die Neuausrichtung der »Verwaltungsrechtswissenschaft als Steuerungswissenschaft « benennt, verfolgt dieser Beitrag das Ziel, den angestrebten Paradigmawechsel selbst zu analysieren. Bei diesem Erkenntnisinteresse gerät nicht nur die Rezeption des Steuerungsbegriffs in den Blickpunkt, sondern - wie bereits erwähnt - vor allem auch jene Trägerschicht, welche das neue Leitbild konzipierte (Invention) und für seine Durchsetzung bei Rechtswissenschaft und Rechtspraxis kämpfte und noch kämpft (Innovation). Dabei wird von der These ausgegangen, dass die Formierung dieser Trägerschicht, die als Sprach- und Diskursgemeinschaft einen eigenen »Begriffshaushalt « kreiert, ${ }^{20}$ selbst das Ergebnis eines kommunikativen Netzwerks ist und die Formierung eines solchen Netzwerks wiederum eine besonders wichtige Voraussetzung für die Formulierung und Durchsetzung des Reform-Leitbilds ist.

Mit dieser Vorgehensweise sind die hiermit vorgelegten Überlegungen insoweit zunächst Thomas S. Kuhn verpflichtet, ${ }^{2 \mathrm{I}}$ als für diesen eine solche Trägerschicht »aus interagierenden Forschungsgruppen mit gemeinsamen Problemen und Zielsetzungen besteht. «22 Die Bezugnahme auf Kuhn gewinnt an Evidenz, wenn man sich - so Ian Hacking - die pragmatische Ausrichtung dieses Forschungsansatzes vor Augen führt:

»Eine derartige (Forscher-)Gruppe ist häufig sogar von einem Außenstehenden zu erkennen, etwa von einem Soziologen, der von dieser Wissenschaft gar nichts versteht. Dieser Nichtfachmann stellt einfach fest, wer mit wem in Verbindung steht, wer am Telefon ist, wer auf

I 8 Hoffmann-Riem, Sozialwissenschaften im Verwaltungsrecht: Kommunikation in einer multi-disziplinären Scientific Community, Die Wissenschaft vom Verwaltungsrecht. Werkstattgespräch aus Anlaß des 60. Geburtstages von Prof. Eberhard Schmidt-Aßmann. Die Verwaltung, Beiheft 2, I999, S. 92.

I9 Hoffmann-Riem, Sozialwissenschaftliche Rechtsanwendung in öffentlich-rechtlichen Übungs- und Prüfungsarbeiten, in: Hoffmann-Riem (Hrsg.), Sozialwissenschaften im Öffentlichen Recht, I98 I, S. 3 ff. (Erster Teil), wo es auf S. I I heißt: »Gefordert ist die intensive Beschäftigung mit Steuerungsfaktoren, die der Entscheidungstheorie geläufig sind. Gemeint sind insbesondere die Faktoren >Personak und >Organisation/Verfahren «.«

20 Vgl. u.a. Stolleis, Wie entsteht ein Wissenschaftszweig? Wirtschaftsrecht und Wirtschaftsverwaltungsrecht nach dem Ersten Weltkrieg, in: Bauer/Czybulka/Kahl/Voßkuhle (Hrsg.), Umwelt, Wirtschaft und Recht. Wissenschaftliches Symposion aus Anlaß des 65. Geburtstages von Reiner Schmidt, 2002, S. I ff.

2 I Kuhn, Die Struktur wissenschaftlicher Revolutionen, I973. Grundlegend zu Kuhn: Hoyningen-Huene, Die Wissenschaftsphilosophie Thomas S. Kuhns. Rekonstruktion und Grundlagenprobleme. Mit einem Geleitwort von Thomas S. Kuhn, I989.

22 Hacking, Einführung in die Philosophie der Naturwissenschaften, 1996, S. 29. Vgl. den Hinweis bei Voßkuhle, Allgemeines Verwaltungs- und Verwaltungsprozessrecht, in: Willoweit (Hrsg.), Rechtswissenschaft und Rechtsliteratur im 20. Jahrhundert, 2007, S. 950: »Maßgebliche Anstöße und Vorarbeiten zu den skizzierten Entwicklungen gehen auf den von Wolfgang Hoffmann-Riem und Eberhard SchmidtAßmann ins Leben gerufenen Reformkreis (!) zurück, der auch methodisch zu einer Neuausrichtung der Verwaltungsrechtswissenschaft geführt hat.« 
der Versandliste für Vorabdrucke steht, wer zu den zahllosen Fachtagungen eingeladen wird, auf denen neueste Forschungsinformationen schon Jahre vor ihrer Veröffentlichung ausgetauscht werden. Ein aufschlußreicher Hinweis sind auch Gemeinsamkeiten in den Literaturhinweisen am Ende der publizierten Aufsätze. «23

Kuhn bietet sich vor allem deshalb an, weil sich sein Konzept des »Paradigmawechsels«bzw. der »wissenschaftlichen Revolution « ${ }^{24}$ nach der von Cohen vorgenommenen Differenzierung in idealtypische Stadien auf die innerhalb der Rechtswissenschaften anzutreffende Unterscheidung zwischen Theorie und Praxis zugeschnitten ist, auch wenn dies von Cohen so nicht intendiert war. ${ }^{25}$

\section{Von der "Revolution im Kopf « zur Verbreitung und Anwendung des Ersonnenen}

Nimmt man Cohens idealtypisch konstruierte Stadien zum Maßstab, so steht am Anfang die »schöpferische Tat« eines Einzelnen oder einer Gruppe, die auf eine Neuausrichtung des bisherigen Verwaltungsrechts abzielt, ohne die in der Disziplin geltenden Standards zu verletzen. Cohen nennt dieses Stadium »Revolution im Kopf.$^{26}$ Herkömmlicher Weise spricht man von einem Einfall, der auf irgendeine Weise zu Papier gebracht werden muss, soll ein Geistesblitz resp. eine Idee nicht in Vergessenheit geraten. Für Cohen beginnt mit der Niederschrift des Ersonnenen das zweite Stadium, ${ }^{27}$ wobei die hierbei mögliche Bandbreite vom Eintrag in ein Tage- oder Notizbuch über eine Skizze (auch zur Aufstellung eines Forschungsprogramms) bis hin zu einer Abhandlung reicht, die wiederum zur Grundlage eines Forschungsantrags werden kann, der nach Bewilligung beispielsweise über die Abfolge von Kolloquien eingelöst wird. Was sich bereits in diesem Stadium abzeichnet bzw. abzuzeichnen beginnt, ist die Einführung einer neuen Terminologie, eines »Bündels von Begriffen, $\aleph^{28}$ möglicherweise sogar im

23 Hacking (Fn. 22), S. 29. Insofern bietet sich zur Erfassung der internen Kommunikationsstruktur als geeignetes Forschungsinstrument die Methode der Zitations- und Co-Zitationsanalyse an, welche sich bei Studien zur Emigration nach 1933 bereits bewährt hat. Die methodologischen und kommunikationstheoretischen Voraussetzungen dieser Methode können hier im Detail nicht erörtert werden, doch sei festgehalten, dass die Verwendung von Zitationsmaßen sich von der Annahme leiten lässt, dass »sich die Beteiligten im Vorgang des förmlichen Zitierens in für die Fachöffentlichkeit sichtbarer Weise wechselseitig wissenschaftliche Anerkennung und fachliche Positionen zuweisen. Vgl. Breunung, Wissenschaftsgeschichte auf dem statistischen Prüfstand. Erkenntnismöglichkeiten quantifizierender Methoden am Beispiel der rechtswissenschaftlichen Amtsvertreibung und Emigration nach 1933, KritV 80 (1997), S. 370 . D. h. ein Autor versucht sich mit seiner "Zitatenwahl in soziale bzw. kognitive Verbindung mit (solchen) Autoren zu bringen, von denen er annimmt, dass sie in der Fachgemeinschaft als qualifizierte Wissenschaftler gelten.«(Breunung, ebd. S. 37I). Über die Verteilung der Bezugnahmen auf Autoren/ Publikationen (Zitationen) sowie über die »durch gemeinsames Zitieren mehrerer Personen und Publikationen in ein und demselben Text hergestellten Verbindungen zwischen verschiedenen Autoren und Schriften (Co-Zitationen)« bildet sich ein Netzwerk persönlicher Beziehungen bzw. wechselseitiger Wertschätzungen (auch im Sinne einer Reputationshierarchie) ab, sichtbar wird aber auch die auf der kognitiven Ebene eingegangene »Verpflichtung « auf ein forschungsleitendes Paradigma (wie Steuerung). Der hiermit skizzierten Methode wird gegenüber der Netzwerkanalyse der Vorzug gegeben, weil sich bei ihr keine Zugangsprobleme stellen und der Aufwand sich in Grenzen hält, da weder Befragungen erforderlich, noch Interaktions- und Austauschbeziehungen zu ermitteln sind. Statt vieler: Schneider, Tauschnetzwerke in der Politikentwicklung. Chemikalienkontrolle in der OECD, EG und der Bundesrepublik Deutschland, Journal für Sozialforschung 26 (I986), S. 383 ff.; Jansen, Einführung in die Netzwerkanalyse, 2003 , S. 69 ff. Grundsätzliches zu der hier vorgestellten Methode: Breunung, Analysen der Wissenschaftsemigration nach 1933. Soziologische und methodologische Überlegungen zum Fall der deutschen Rechtswissenschaft, Zeitschrift für Soziologie 25 (I996), S. $395 \mathrm{ff}$.

24 Vgl. Hoyningen-Huene (Fn. 21), insb. Kap. 4: »Der Paradigmabegriff«, S. I 33 ff., sowie Kap. 6: »Der Begriff der wissenschaftlichen Revolution«, S. I93 ff.

25 Cohen, Revolutionen in der Naturwissenschaft, 1994.

26 Cohen (Fn. 25), S. 65.

27 Cohen (Fn. 25), S. 65 f.

28 Cohen (Fn. 25 ), S. 65 . 
Lichte eines besonders herausgestellten Leitgedankens, wodurch bisherige Denkgewohnheiten in Frage gestellt werden (können).

Wer Kolloquien organisiert, macht den nächsten Schritt in die disziplinäre (Bereichs-)Öffentlichkeit. In diesem dritten Stadium geht es um Vertiefung und Verbreitung des schriftlich festgehaltenen Reformkonzepts unter Mitarbeitern, Schülern und Kollegen - vor allem unter solchen Kollegen, die dem Vorhaben Unterstützung geben können, sei es, weil sie ähnliche Vorstellungen haben, sei es, weil sie bei der Disziplin hohes Ansehen genießen. Am Ausmaß der auf den Kolloquien erfahrbaren Unterstützung durch Kollegen sowie an ersten Reaktionen auf die abgehaltenen Tagungen lässt sich zunächst einmal ablesen, inwieweit einzelne Kollegen bereit sind, dem Reformvorhaben durch engagierte Unterstützung Aussicht auf Erfolg einzuräumen. In diesem Stadium der erstmaligen Verbreitung der Reformüberlegungen setzt sich das ganze Projekt auch der erstmaligen Fachkritik aus. Dabei ist nicht zu übersehen, dass in diesem frühen Stadium zwischen Unterstützung und Kritik ein prekäres Spannungsverhältnis besteht: Wer auf Unterstützung aus ist, verzichtet gerne auf Kritik, wie bei grundsätzlicher Kritik gar mit Ablehnung des Reformvorhabens zu rechnen ist. Bereichsöffentlichkeit lässt sich über erprobte Einrichtungen des Wissenschaftsbetriebs leicht herstellen: über Fachbereichskolloquien oder wissenschaftliche Tagungen, mit denen üblicherweise bestimmte Publikationsformen einhergehen, wie preprints, working- oder discussion papers, die ihrerseits wissenschaftlichen Abhandlungen in Fachzeitschriften vorausgehen und Neugier wecken sollen. Für Cohen ist dieses (idealtypisch konstruierte) dritte Stadium, das er unter ausdrücklicher Anspielung auf eine Formulierung in Galileis »Dialog über die beiden hauptsächlichen Weltsysteme« (1632) als »Revolution auf dem Papier« kennzeichnet, ein Übergangsstadium, bei dem »eine Idee oder ein Bündel von Ideen die Schwelle zur allgemeinen Zirkulation unter den Mitgliedern der scientific community überschreiten. $\ll^{29}$

Allerdings kann ein intendierter Paradigmawechsel »in jedem dieser drei ersten Stadien fehlschlagen. «3० Solange hinter dem neuen Paradigma keine größere Trägerschicht engagierter Wissenschaftler steht, muss damit gerechnet werden, dass der intendierte Paradigmawechsel eine »Revolution auf dem Papier«bleibt. Um zunächst innerhalb des Wissenschaftsbetriebs den beabsichtigten Paradigmawechsel auch tatsächlich herbeizuführen, ist es erforderlich, dass in einem vierten Stadium sich eine genügende Anzahl auch jüngerer Wissenschaftler entschließt, die eigene Arbeit an dem neuen Paradigma auszurichten, d. h. diese Wissenschaftler übernehmen in ihren Publikationen die neue Terminologie und nehmen dabei wechselseitig aufeinander Bezug. ${ }^{3 \mathrm{I}}$ Es ist ohne weiteres einsichtig, dass die Formierung eines dem neuen Paradigma verpflichteten Netzwerks einer Trägerschicht - eine wichtige Voraussetzung dafür bildet, dass die »wissenschaftliche Revolution« weiter fortschreitet und tiefer in die Disziplin hinein getragen wird. Die »erstmalige « Ankunft dort ist zu vermelden, wenn beispielsweise »in Lehrbüchern des Öffentlichen Rechts (...) die (neuen) Sichtweisen « erstmals registriert werden ${ }^{2}$ bzw. die Vereinigung der Staatsrechtslehrer auf

29 Cohen (Fn. 25), S. 67. Die nämliche Textstelle aus Galileis »Dialog« lautet: »Unsere Untersuchungen haben die Welt der Sinne zum Gegenstand, nicht eine Welt von Papier.« Zitiert nach Cohen, ebd., S. 67, Fn. 3. 30 Cohen (Fn. 25), S. 69.

3I Cohen (Fn. 25), S. 70 f.

32 Hoffmann-Riem (Fn. I 8), S. 93. "Erstmals « ist als Hinweis darauf zu verstehen, dass mit der Registrierung in Lehrbüchern hiermit noch keineswegs der Zustand der »normalen Wissenschaft « erreicht ist, zu der ein neues Paradigma in der Regel wird, wenn es weitestgehende Anerkennung im Wissenschaftsbetrieb findet. 
ihren Jahrestagungen sich mit dem neuen Paradigma auseinander setzt und dieses dadurch, wenn auch oftmals nur in Teilaspekten, »juristisch >geadelt « « wird..$^{33}$ Auch die auf die dort gehaltenen Vorträge folgende Diskussion gibt Hinweise auf Ablehnung bzw. Akzeptanz von Grundannahmen des neuen Paradigmas vor einem ausgewiesenen Fachpublikum, zumal solche Grundannahmen kontrovers vorgestellt und gewürdigt werden. Andererseits lassen sich über Vorträge oder Diskussionsbeiträge leichter geeignete Kandidaten zur Unterstützung des neuen Paradigmas gewinnen, vor allem dann, wenn für dieses in der durchaus kontrovers geführten Aussprache in der Reputationshierarchie hoch angesiedelte, zumal interdisziplinär aufgeschlossene Fachvertreter eintreten. 34 Bei diesen handelt es sich im vorliegenden Fall nicht um »von Skepsis gegenüber der eigenen Disziplin geplagte Außenseiter, die disziplinäre Grenzüberschreitungen gewagt und erfolgreich praktiziert haben. «35

Gerade mit Blick auf die hier interessierende juristische Disziplin, die sich durch eine interne Differenzierung zwischen Wissenschaft und Praxis (Rechtsprechung, Gesetzgebung) auszeichnet, ${ }^{36}$ ist strikt zwischen Invention und Innovation zu trennen. 37 Aus der Invention eines (neuen) Paradigmas, das innerhalb des Wissenschaftsbetriebs Beachtung, wenn nicht gar Anerkennung bei der tonangebenden disziplinären Bereichsöffentlichkeit findet (wie sie von der Vereinigung der Staatsrechtslehrer repräsentiert wird), wird erst eine Innovation, wenn sich auch Rechtsprechung und/oder Gesetzgebung an diesem Paradigma orientieren..$^{3}$ Dies gilt für den Fall, dass die Invention auf Seiten der theoretischen Rechtswissenschaft, also innerhalb des Wissenschaftsbetriebs, geboren wird.39 Dass der Wissenschaftsbetrieb als »Geburtsstätte « von Inventionen gilt, ist nicht ungewöhnlich, zumal der Wissenschaft allgemein schöpferisches Potential zugeschrieben wird. Im konkreten Fall ist die intendierte Reform des Verwaltungsrechts explizit als ein »Projekt der Wissenschaft « verstanden und betrieben worden, bei dem durch den »weitgehende(n) Verzicht auf die Beteiligung von

33 Hoffmann-Riem (Fn. I8), S. 93. Siehe die Tagung 1996 in Dresden zum Thema »Verwaltung und Verwaltungsrecht zwischen gesellschaftlicher Selbstregulierung und staatlicher Steuerung «, VVDStRL 56 (1997), S. I62 ff. (Referat Schmidt-Preuß) sowie VVDStRL 56 (I997), S. 235 ff. (Referat Di Fabio).

34 Beispielhaft hierfür sind auf der Dresdner Tagung die Diskussionsbeiträge von Hoffmann-Riem, Schmidt-Aßmann und Schuppert, siehe VVDStRL 56 (I997), S. 29I ff. Bezeichnend auch, dass der erste Angriff auf ein neues Paradigma der mit ihm verbundenen neuen Terminologie gilt, dem sinnfälligsten Zeichen für eine intendierte Neuausrichtung. So Ossenbühl als erster Diskussionsredner, siehe VVDStRL 56 (I997), S. 283 f.

35 Czada, Disziplinäre Identität als Voraussetzung von Interdisziplinarität?, in: Bizer/Führ/Hüttig (Hrsg.), Responsive Regulierung. Beiträge zur interdisziplinären Institutionenanalyse und Gesetzesfolgenabschätzung, 2002, S. 30.

36 Bei Schmidt-Aßmann (Zur Situation der rechtswissenschaftlichen Forschung. Eine Gedankenskizze, JZ 5० (I995), S. 7) kehrt diese Unterscheidung wieder mit der Differenzierung zwischen »Forschungssystem « und »Entscheidungssystem.«

37 Schulze-Fielitz (Was macht die Qualität öffentlich-rechtlicher Forschung aus?, Jahrbuch des Öffentlichen Rechts der Gegenwart 50 (2002), S. 42) nimmt in seinem informativen Beitrag diese Unterscheidung nicht vor, obwohl er sieht, dass die Durchsetzung einer Invention (bei Schulze-Fielitz: Innovation) »in Wissenschaft oder Praxis von in weitestem Sinne rechtskulturellen Rahmenbedingungen abhängt.«

38 Siehe auch den auf die Naturwissenschaften gemünzten Hinweis bei Knorr-Cetina, Die Fabrikation von Erkenntnis: Zur Anthropologie der Naturwissenschaft, 2004, S. I 23: »Aber die Schöpfung einer Metapher oder die Herstellung einer Analogie ist nicht als solche selbst bereits ein Stück >wissenschaftlicher Innovation $\prec$ Forschungsprodukte, die als wissenschaftliche Innovationen klassifiziert werden wollen, müssen ein entscheidendes Element materiell und interpersonell erzeugten Erfolgs aufweisen: Erfolg bei der Konstruktion im Labor, Erfolg bei der Annahme durch andere Wissenschaftler und schließlich Erfolg beim Überzeugen der Historiker, daß das Produkt in der Tat eine >Innovation< darstellt."

39 So hat das BVerfG beispielsweise in einem Urteil erkannt, dass »sich die Regelungen des Bundes-Immissionsschutzrechts zur Konkretisierung der abfallrechtlichen Pflichten als >billigende Programmierung von kooperativem Verwaltungshandeln dar(stellen) - mit Hinweis auf Dose/Voigt (Hrsg.), Kooperatives Recht, I995, S. 17. Siehe NJW i 998 , S. 2348. 
Praktikern «4० offensichtlich ein erheblich höheres Reformniveau angestrebt wurde, wird doch der Praxis üblicherweise unterstellt, sie sei in der Regel nur zu inkrementalen Reformen fähig.

Eine solche Vorgehensweise hat in besonderer Weise dem Umstand Rechnung zu tragen, dass die institutionell verankerte Zweiteilung der Rechtswissenschaft in Theorie und Praxis den Erfindern eines neuen Paradigmas die Fähigkeit zu »Umweghandlungen « zugunsten der Funktionslogik einer (autonomen) Rechtspraxis abverlangt, eine Fähigkeit, auf welcher nach Popitz Innovationen letztlich zu beruhen scheinen. ${ }^{4}$ Eine »als Projekt der Wissenschaft « betriebene Neuausrichtung des Verwaltungsrechts, die mit der Anleihe bzw. Übernahme einer fachfremden Terminologie darauf setzt, dass sie dadurch erheblich ihre Analysefähigkeit stärkt, weil sie Zweifel hat, »ob die bisherige Begrifflichkeit die Wirklichkeit, und zwar die normative und die normgeprägte Wirklichkeit, hinreichend in den Blick bekommt, ${ }^{2}{ }^{2}$ hat sich auf der anderen Seite jedoch $\mathrm{zu}$ vergewissern, dass sie die erforderliche »Rückbindung an die Rechtsdogmatik « vornimmt. Wenn ausgewiesene Fachleute hier Defizite konstatieren,43 so ist andererseits ein informierter » Wirklichkeitsbezug « dadurch gewährleistet, dass die theoretische Rechtswissenschaft schon immer "am ehesten Impulse der Praxis aufgegriffen (hat), die zuvor in innovationsgeneigten Referenzfeldern (wie Umwelt-, Informations- und Technikrecht) praktisch eingesetzt worden sind.«44 Freilich soll mit der Abkehr von den traditionellen Referenzgebieten (Bau-, Polizei-, Kommunal- und Beamtenrecht), die in der Gerichtspraxis und Kommentarliteratur »vorrangig eine Rolle spielen « und infolgedessen »ihre gebietstypischen Erfahrungen, Lösungselemente und Anforderungen in die Dogmatik des allgemeinen Verwaltungsrechts « einbringen, und mit der stärkeren Hinwendung auf die »innovationsgeneigten Referenzfelder « die bisherige »(Verwaltungs-)Gerichtslastigkeit« mit ihrer Ausrichtung an »gesetzesvollziehende(n) Einzelentscheidung(en)«45 reduziert werden zugunsten einer Erfassung der »vielfältige(n) Beziehungen und Verflechtungen zwischen staatlichen und gesellschaftlichen, privaten und öffentlichen Akteuren, « wofür das von den Sozialwissenschaften entlehnte »analytische Konzept « der Steuerung ${ }^{46}$ mit der besonderen Aufmerksamkeit für »Vorgänge kooperativen und mittelbaren Bewirkens« einstehen soll.47 Dennoch ist, so einschränkend Hoffmann-Riem, nicht auszuschließen, dass die mit einer solchen Perspektivenerweiterung ver-

40 Voßkuhle, Die Reform des Verwaltungsrechts als Projekt der Wissenschaft, DV 32 (I999), S. 553; ferner Schmidt, Die Reform von Verwaltung und Verwaltungsrecht. Reformbedarf - Reformanstöße - Reformdebatte, Verwaltungsarchiv 9i (2000), S. I 49 ff.

4I Popitz, Epochen der Technikgeschichte, 1989, S. i4 f.

42 Diskussionsbeitrag von Hoffmann-Riem auf der Dresdner Tagung der Staatsrechtslehrer. Siehe VVDStRL 56 (1997), S. 29 I.

43 Möllers, Theorie, Praxis und Interdisziplinarität in der Verwaltungsrechtswissenschaft, Verwaltungsarchiv 93 (2002), S. 3 I u. S. 43 ff. Ferner: Röhl, Verwaltungsverantwortung als dogmatischer Begriff?, Die Wissenschaft vom Verwaltungsrecht. Die Verwaltung, Beiheft 2, I999, S. 33 ff., sowie Schulte, Wandel der Handlungsformen der Verwaltung und der Handlungsformenlehre in der Informationsgesellschaft, in: Hoffmann-Riem/Schmidt-Aßmann (Hrsg.), Verwaltungsrecht in der Informationsgesellschaft, 2000, S. 346 f. Siehe auch Lepsius, Steuerungsdiskussion, Systemtheorie und Parlamentarismuskritik, I999, S. 7 f. Ferner: Wahl, Herausforderungen und Antworten: Das Öffentliche Recht der letzten fünf Jahrzehnte, 2006, insb. S. $87 \mathrm{ff}$.

44 Hoffmann-Riem, Risiko- und Innovationsrecht im Verbund, DV 38 (2005), S. I74.

45 Schmidt-Aßmann, Zur Reform des Allgemeinen Verwaltungsrechts - Reformbedarf und Reformansätze, in: Hoffmann-Riem/Schmidt-Aßmann/Schuppert (Hrsg.), Reform des Allgemeinen Verwaltungsrechts. Grundfragen, I993, S. I4 ff.; Schmidt-Aßmann (Fn. I6), S. I 48 f.

46 Schmidt-Aßmann, Das Allgemeine Verwaltungsrecht als Ordnungsidee. Grundlagen und Aufgaben verwaltungsrechtlicher Systembildung, I998, S. I9 ff.

47 Schmidt-Aßmann (Fn. 45), S. I6. 
keit« zu reduzieren ist, wie auch »die Komplexität der Lebensverhältnisse (...) etwa durch Segmentierung und Anknüpfung der rechtlichen Steuerung an identifizierbare Einzelakte oder Akteure « gewöhnlich reduziert wird. $4^{8}$

Wenn die kursiv hervorgehobene Formulierung auf »Vorgänge kooperativen und mittelbaren Bewirkens « abzielt, werden damit erste Hinweise auf disziplinäre Grenzüberschreitungen gegeben, deren Möglichkeiten bei den von der juristischen Disziplin vorgegebenen Rahmenbedingungen im folgenden Abschnitt erörtert werden. Zum einen kommt das die damalige Diskussion beherrschende Leitbild des »kooperativen Staates « ins Blickfeld, dessen Funktionsweise wie dem Recht dabei eingeräumten Steuerungschancen relativ früh durch von Ritter (einem Praktiker) vorgelegte Veröffentlichungen erschlossen wurden,49 zum anderen ein von R. Mayntz I 987 offeriertes Steuerungsmodell, auf das noch ausführlich eingegangen wird.

\section{Zur relativen "Geschlossenheit" der juristischen Disziplin und den sich bieraus ergebenden Konsequenzen}

Es ist zuallererst daran zu erinnern, dass es sich bei den Rechtswissenschaften um eine normative Wissenschaft handelt. Dies wirft die Frage auf, welche Austauschformen bzw. Transfermöglichkeiten mit anderen, zumal sich als empirisch-analytisch verstehenden Wissenschaften eine normative Wissenschaft überhaupt zulässt, die ihre Identität aus einem anerkannten Lehrkanon (Dogmatik) und einem feststehenden Methodenrepertoire bezieht, ${ }^{\circ}$ sich ferner durch eingeübte Denkgewohnheiten und Argumentationsstile (h. L.) auszeichnet, d. h. durch ein hohes Ausmaß an »Schließung nach außen« (Max Weber) gekennzeichnet ist.

Die eben angesprochene Problematik lässt sich so angehen, dass mögliche Formen disziplinüberschreitender Kommunikation nach dem Grad ihrer Intensität der wissenschaftlichen Kommunikation und der hierbei bevorzugten Richtung unterschieden werden. So charakterisiert den Pol mit der geringsten Intensität und einer unidirektionalen Gerichtetheit diejenige Form, die sich darauf beschränkt, dass sich eine Disziplin im Hinblick auf abgegrenzte Problembereiche (Themengebiete) von ausgewählten Fachvertretern (Experten) einer anderen Disziplin über die dort üblichen fachspezifischen Betrachtungs- und Vorgehensweisen (Methoden) informieren lässt, was dazu führt, dass beide Seiten die gegebenen Disziplingrenzen ausdrücklich wechselseitig bestätigen. Diese der Perspektivenerweiterung dienende Kommunikationsform, von R. Czada mit dem Etikett der »Multidisziplinarität« versehen, ist für den »herkömmlichen Umgang (...) der Rechtswissenschaften « mit Nachbardisziplinen, die dadurch den Status als »Hilfswissenschaften « zugewiesen bekommen, typisch..$^{\text {I }}$

\footnotetext{
48 Hoffmann-Riem (Fn. 44), S. I55.

49 Ritter, Der kooperative Staat. Bemerkungen zum Verhältnis von Staat und Wirtschaft, Archiv des öffentlichen Rechts I04 (I979), S. 389 ff.; Ritter, Das Recht als Steuerungsmedium im kooperativen Staat, Staatswissenschaften und Staatspraxis I ( I990), S. 50 ff. Es ist kein Zufall, dass kooperative Beziehungen beim Umweltschutz empirisch nachgewiesen wurden, so dass dieser nicht von ungefähr den Status eines modernen Referenzgebietes erhielt. Statt vieler: Trute, Vom Obrigkeitsstaat zur Kooperation. Zur Entwicklung des umweltrechtlichen Instrumentariums zwischen klassischem Ordnungsrecht und moderner Verwaltung, Rückzug des Ordnungsrechtes im Umweltschutz, I999, S. I3 ff.

50 Schulte (Fn. 43), S. 343 f.

s I Czada (Fn. 35), S. 25 f. Die hier angewandten begrifflichen Unterscheidungen von »Multidisziplinarität « und »Interdisziplinarität« decken sich nicht mit jenen, die Schmidt-Aßmann ([Fn. 36], S. 7 ff.) heranzieht.
} 
Den Gegenpol hierzu bietet eine Austauschform, die auf die »Übertragung von Methoden oder Theoriebausteinen aus einer Disziplin in eine andre « abstellt und die, so Czada, allein die Bezeichnung »interdisziplinär « verdient. ${ }^{2}$ Wie Czada weiter ausführt, kann auf einer erkenntnistheoretischen Ebene Interdisziplinarität auch bei der nach außen relativ geschlossenen juristischen Disziplin zur Übernahme von Theorien führen, was sich mit Hinweisen auf die »Ökonomische Theorie des Rechts«, die »Neue Institutionenökonomik « oder die Spieltheorie belegen lässt. 53 Häufig führt jedoch, so ebenfalls Czada, die »Übernahme kognitiver oder methodologischer Elemente aus anderen Disziplinen lediglich zur Anreicherung eigener Erklärungsansätze oder allenfalls zur Herausbildung von Subdisziplinen « am Rand der Mutterdisziplin.54 Zwischen diesen beiden Polen bewegen sich Transferleistungen, die in unterschiedlichem Maße einem Erkenntnisgewinn bzw. einer Perspektivenerweiterung dienen (können). 55

Wie bereits ein erster Blick auf die Publikationen zeigt, die aus den (in Hamburg und Heidelberg) durchgeführten Tagungen, auf denen die »Reform des Verwaltungsrechts « angestoßen bzw. vorangetrieben wurde, hervorgegangen sind, 56 wurde die Reformdebatte mit schulenbildender Wirkung (»Schließung nach innen«) keineswegs als multidisziplinärer Dialog organisiert, bei dem »Vertreter unterschiedlicher Disziplinen zusammentreffen und ihre Ansichten, Forschungsergebnisse und Problemlösungsvorschläge austauschen. «57 Von Ausnahmen abgesehen, traf sich auf den späteren Tagungen eine eher »geschlossene Gesellschaft« von Vertretern des Öffentlichen Rechts bzw. Verwaltungsrechts (bei einer Tagung wurden Zivilrechtler hinzugezogen), ${ }^{8}$ die sich jedoch alle durch eine Aufgeschlossenheit gegenüber Nachbarwissenschaften (insb. Verwaltungs- und Sozialwissenschaften) auszeichneten. Insofern liegt es nahe zu fragen, auf welche Weise sich die »Aneignung « kognitiver oder methodologischer Elemente aus anderen Disziplinen unter diesen Bedingungen vor allem vollzieht.

\section{I Der »aufgeschlossene Jurist « als »versierter Bastler» (Bricoleur)}

Die meisten der in den Tagungsbänden zur »Reform des Verwaltungsrechts« veröffentlichten Beiträge sowie die hierauf Bezug nehmenden Publikationen sind von Juristen verfasst, die sich gegenüber den Sozialwissenschaften aufgeschlossen zeigen. Diese Juristen zitieren von Sozialwissenschaftlern veröffentlichte Arbeiten, welche die Bevorzugung einer ganz bestimmten Denk- und Forschungsrichtung, die des akteurorientierten Institutionalismus',59 erkennen lassen und mit dieser die Bevorzugung ihrer beiden führenden Repräsentanten, Renate Mayntz und Fritz W. Scharpf, die innerhalb und außerhalb ihrer Disziplin hohes Ansehen genießen. ${ }^{60}$ Es ist nicht zu übersehen, dass die von Sozialwissenschaftlern verfassten Texte höchst selektiv herangezogen werden, d.h. sie

\footnotetext{
52 Czada (Fn. 35), S. 25.

53 Czada (Fn. 35), S. 25.

54 Czada (Fn. 35), S. 26.

55 Hoffmann-Riem (Fn. I 8), S. 92.

56 Voßkuhle (Fn. 40), S. 545 .

57 Czada (Fn. 35), S. 25.

58 Hierbei handelt es sich um die Form der Intradisziplinarität, vgl. Schmidt-Aßmann (Fn. 36), S. 8.

59 Mayntz/Scharpf, Der Ansatz des akteurzentrierten Institutionalismus, in: Mayntz/Scharpf (Hrsg.), Gesellschaftliche Selbstregelung und politische Steuerung, I995, S. 39 ff. Die Bevorzugung dieses Ansatzes kommt freilich eher einem Bekenntnis gleich, da mit den Theoriebausteinen dieses Ansatzes keineswegs argumentiert wird. Als eindrucksvolles Gegenbeispiel: Wiesner, Politik unter Einigungszwang. Eine Analyse föderaler Verhandlungsprozesse, 2006.
} 
werden unter ganz bestimmten, von der juristischen Disziplin vorgegebenen Gesichtspunkten rezipiert, nicht selten beschränkt sich die Anleihe bei den Sozialwissenschaften auf die Übernahme von Fachtermini, die in ihrem anders gearteten Verwendungszusammenhang oft genug eine neue Bedeutung erhalten, deren Übernahme aber auch die Identität als Rechtswissenschaftler bedrohen kann:

»In dem Prozeß sozialer Sinnkonstruktion - als der sowohl die rechtswissenschaftliche Theoriebildung als auch die praktische Rechtsanwendung verstanden werden kann - dominieren im ersten Zugriff die Erfahrungen, Interessen und Handlungszwänge der Verwender des Wissens. Ihre Erfahrungen werden aber durch den Umgang mit der je anderen Wissenschaft selbst geprägt. Die Rechtswissenschaft läßt sich auf die Sprachwelt der Sozialwissenschaft ein - auch wenn sie die fachsprachliche Spezialisierung kritisiert (...).« Mit dem eben angesprochenen Zugriff auf die Sozialwissenschaft durchläuft diese, »wenn sie in Lehre und/ oder Praxis folgenreich werden will, Filter einer spezifischen Verwendungstauglichkeit, «was aus Sicht der sozialwissenschaftlichen Fachvertreter zu einer Einbuße ihres Erklärungspotentials führe, die soweit gehen kann, dass sogar von einer »Banalisierung und Trivialisierung « gesprochen werden könne. ${ }^{61}$

Nach dem bisher Ausgeführten ist festzuhalten, dass die den »Filter der Verwendungstauglichkeit « durchlaufende Rezeption durch aufgeschlossene Juristen keine echte Austauschbeziehung darstellt, zumal die Übertragungsrichtung einseitig ist (also eine unidirektionale Gerichtetheit aufweist). Auch kann nicht von »Multidisziplinarität « gesprochen werden, auch wenn die »Verwaltung « selbst einen multidisziplinären Untersuchungsgegenstand darstellt.

Die eben mitgeteilten Befunde sind eine Folge der »innerjuristischen Verhältnisse« (Max Weber), die sich u.a. mit Hilfe des Begriffspaars der »funktionale(n)« und »strategische(n) Abhängigkeit der Disziplinangehörigen untereinander « näher erfassen lassen. Bezieht sich die zuerst genannte Form interner Abhängigkeit »auf gemeinsame Kompetenz-Standards, Denk- und Argumentationsstile, die Anwendung ähnlicher Methoden (...), « so verweist die zweite Form auf das Ausmaß an »disziplinbezogener Identität « und »professioneller Geschlossenheit «, die ja per se Abgrenzung bzw. Grenzziehung bedeutet. ${ }^{62}$ So gesehen zeichnen sich die Rechtswissenschaften durch eine hohe interne funktionale wie hohe interne strategische Interdependenz aus. Hiermit hängt zusammen, dass es sich bei den Rechtswissenschaften um eine normative Disziplin handelt, welche nicht nur Ordnungsstrukturen entwirft, welche die » Wirklichkeit « normativ erfassen, sondern auch infolge ihrer Gebundenheit an Recht und Gesetz sich nur in begrenztem Umfang auf andere Disziplinen einlassen kann. ${ }^{63}$ Und wenn sie dies tun, geschieht dies vornehmlich »nur in einer Richtung «, was darauf zurückzuführen ist, dass eine solche »Membrandisziplin «(Czada) infolge ihrer hohen internen funktionalen Abhängigkeit der Disziplinangehörigen untereinander über ein relativ »kohärentes kognitives Angebot « in Gestalt der abrufbaren Dogmatik verfügt, ${ }^{64}$ mit deren Hilfe sie »passend erscheinende $^{4}$ Angebote anderer Disziplinen »filtern« kann, zumal dann, wenn sich diese

60 Vgl. Klingemann/Falter, Die deutsche Politikwissenschaft im Urteil der Fachvertreter. Erste Ergebnisse einer Umfrage von 1996/1997, in: Greven (Hrsg.), Demokratie - eine Kultur des Westens?, 20. Wissenschaftlicher Kongreß der Deutschen Vereinigung für Politische Wissenschaft, 1998, S. 327, S. 333 , S. 335 .

6r Hoffmann-Riem (Fn. i 8), S. 85 f., eigene Hervorhebung.

62 Czada (Fn. 35), S. 36 f. Das Begriffspaar der »funktionalen« und »strategischen Abhängigkeit « verdankt sich Czada. Ein hohes Ausmaß an »disziplinbezogener Identität« und »professioneller Geschlossenheit« korrelieren miteinander.

63 Czada (Fn. 35), S. 47 f. Siehe ferner Schmidt-Aßmann, Gesetzes- und Rechtsbindung der Verwaltung, in: König/Siedentopf (Hrsg.), Öffentliche Verwaltung in Deutschland, I 996/97, S. 359 ff.

64 Czada (Fn. 35), S. 36. 
Angebote auf solche Felder erstrecken, die dem Verwaltungsrecht zugleich als »moderne Referenzgebiete « gelten.

Solchermaßen skizzierte Rahmenbedingungen begünstigen ein einseitiges Rezeptionsverhalten durch aufgeschlossene Juristen, ${ }^{65}$ das sich unter Bezugnahme auf Claude Lévi-Strauss als intellektuelle »Bastelei « (bricolage) bezeichnen lässt. ${ }^{66}$ Der aufgeschlossene Jurist repräsentiert insofern den Typus des »versierten Bastlers«, als er auf ihn interessierende Disziplinen einen »instrumentellen Zugriff « praktiziert, d. h. die mögliche Übernahme kognitiver und/ oder methodologischer Elemente bzw. Wissensbestände von anderen Disziplinen »nach dem Prinzip, >das kann man immer noch brauchen« «67 zusammen trägt und entsprechend auswählt. D. h. Texte anderer Disziplinen werden, um die Sprache des Bastlers zu bemühen, »ausgeschlachtet «, um die so identifizierten Teilstücke möglichst im Lichte eines »Ordnungskonzepts « oder, falls ein solches nicht vorhanden ist bzw. erst entwickelt werden muss, im Lichte einer Leitidee zusammenzusetzen. Der versierte Bastler zeichnet sich also dadurch aus, dass er einen begrenzten Bestand an verfügbaren »Mitteln « durch Angebote von anderen Disziplinen erweitert, indem er beispielsweise sog. »Schlüsselbegriffe« erfindet und die Liste solcher und ähnlicher Begriffe bedarfsgerecht ergänzt. Hierzu zählt auch die besondere Fähigkeit, mit Hilfe metaphorischer Übertragung »bestehende Kategorisierungen aufzubrechen und neue wissenschaftliche Begriffe zu konstituieren. ${ }^{68}$ Neben dieser anspruchsvollen Form der »bricolage« gibt es allerdings auch weniger anspruchsvolle Bastelarbeiten, bei denen mit »Begriffen « so umgegangen wird wie mit den Klötzchen eines Baukastens. Um »vorhandene funktionsspezifische Ansätze unterschiedlicher Wissenschaftsdisziplinen oder Teildisziplinen fruchtbar $\mathrm{zu}$ machen, ${ }^{69}$ ist beispielsweise » Die Verwaltung als ...<-Methode« erfunden worden, mit deren Hilfe dann die nachstehend aufgeführten »vier Funktionsbereiche der Verwaltung « gewonnen wurden: »Die öffentliche Verwaltung als Organisation, - als Entscheidungssystem, - als öffentlicher Dienst, - als Unternehmen. « Mit diesen vier Funktionsbereichen seien »zugleich die vier wichtigsten Steuerungsebenen des Verwaltungshandelns « mitgegeben, nämlich: »Steuerung durch Entscheidungsprogramme, - durch Organisation und Verfahren, - durch Personal, durch Finanzen und Haushalt, « was »als funktionenübergreifende Perspektive

65 Zum Rezeptionsverhalten der juristischen Disziplin siehe Eifert, Innovationen in und durch Netzwerkorganisationen: Relevanz, Regulierung und staatliche Einbindung, in: Eifert/Hoffmann-Riem (Hrsg.), Innovation und rechtliche Regulierung. Schlüsselbegriffe und Anwendungsbeispiele rechtswissenschaftlicher Innovationsforschung, 2002, S. Iо Iff.; sowie Bumke, Die Entwicklung der verwaltungswissenschaftlichen Methodik in der Bundesrepublik Deutschland, in: Schmidt-Aßmann/Hoffmann-Riem (Hrsg.), Methoden der Verwaltungsrechtswissenschaft, 2004, S. I03 ff., S. I Is ff.

66 Lévi-Strauss, Das wilde Denken, I98 I, S. 29 ff. Siehe auch Rheinberger, Experimentalsysteme und epistemische Dinge. Eine Geschichte der Proteinsynthese im Reagenzglas, 200 I, S. 24 ff., S. 244 ff.; sowie Treiber, Trägt die Vorschriftenbereinigung zum effektiven Vollzug bei? Oder die Entdeckung des versierten »Bastlers « im Vorschriftendschungel, KJ 25 (1992), S. 32 ff. Dass mit dieser Kennzeichnung keineswegs eine negative Bewertung verbunden ist, mag mit einem Zitat aus Rheinberger (ebd., S. 30) unterstrichen werden: »Wissenschaftler sind vor allem `Bastler«, Bricoleure (...). « Auch Lévi-Strauss verbindet mit dem Ausdruck »Bastler keine herabsetzende Bewertung.

67 Lévi-Strauss (Fn. 66), S. 30.

68 Drewer, Die kognitive Metapher als Werkzeug des Denkens. Zur Rolle der Analogie bei der Gewinnung und Vermittlung wissenschaftlicher Erkenntnisse, 2003, S. 60. (Kritisch) rezensiert durch N. Janich, Muttersprache i is (2005), S. 8 I ff. Zu Drewer sowie zu einigen anderen wichtigen Metaphern-Studien werden Rezensionen benannt, weil diese auf anschauliche Weise die auf bestimmte Theorierichtungen bzw. Disziplinzugehörigkeiten rückführbaren unterschiedlichen Positionen anzeigen.

69 Schuppert, Soziologie der öffentlichen Verwaltung. Aufgaben einer zeitgemäßen Verwaltungssoziologie, in: Dreier (Hrsg.), Rechtssoziologie am Ende des 20. Jahrhunderts. Gedächtnissymposion für Edgar Michael Wenz, 2000, S. 2 I I f. 
einen steuerungstheoretischen Ansatz « hervorbringe. Genau besehen handelt es sich weder durchgehend um Funktionsbereiche, ${ }^{70}$ noch sind die vier Funktionsbereiche von den vier Steuerungsebenen trennscharf voneinander unterschieden, noch kann auf die vorgegebene Weise ein »steuerungstheoretischer Ansatz« gewonnen werden, wie es überhaupt einer theoretisch fundierten Begründung für die vier proklamierten »Funktionsbereiche« der Verwaltung bedurft hätte.

Bei den gegebenen Restriktionen disziplinüberschreitender Kommunikation erscheint die Erfindung der sog. »Schlüsselbegriffe« im allgemeinen und des Schlüsselbegriffs der »Steuerung « im besonderen als eine dem »Ei des Kolumbus « vergleichbare Lösung, mit der nunmehr ein Vergleichspunkt existiere, der "sich von den jeweiligen Fachterminologien befreit hat und als anschlussfähiger Referenzpunkt für die Reformulierung der Aussagen der jeweiligen Disziplinen dient. «7I Mit dem so verstandenen Schlüsselbegriff der »Steuerung « wurde in den Augen der Reformjuristen nicht nur das Tor zu den Sozial- bzw. Verwaltungswissenschaften aufgeschlossen, sondern auch die Neuausrichtung der »Verwaltungsrechtswissenschaft als Steuerungswissenschaft« ermöglicht.

\section{Von der Dogmatik zur Steuerungswissenschaft $7^{2}$}

Auf einer Tagung Ende November i99I in Hamburg »entwarf G.F. Schuppert Konturen einer >Verwaltungsrechtswissenschaft als Steuerungswissenschaft $<$, « bei der »die Verwaltungswissenschaft zur Grundlage der juristischen Begriffsund Systembildung würde.«73 Mit dieser Vorstellung vertrat er damals eine Gegenposition zu E. Schmidt-Aßmann, der »das allgemeine Verwaltungsrecht (...) weiterhin als eine systematische Disziplin « verstand, 74 jedoch dieses - »in einer Verbindung von theoretischer und praktischer Sichtweise «, von deduktiver und induktiver Vorgehensweise - der verwaltungswissenschaftlichen Forschung annähern wollte, der dadurch - anders als bei Schuppert - eine bloße Korrektur- und Ergänzungsfunktion zugewiesen wurde.75 Indem das allgemeine Verwaltungsrecht sich einerseits an den normativen Vorgaben der »Verfassungsstrukturentscheidungen « des Grundgesetzes für Rechtsstaat, Demokratie und offene Staatlichkeit auszurichten hat, ${ }^{76}$ andererseits »empirisch « gesättigt wird durch "prägende Vorbildbereiche des Besonderen Verwaltungsrechts «,77 d. h. durch sog. Referenzgebiete, erhalten die Verwaltungs- bzw. Sozialwissenschaften genau besehen sogar eine geschmälerte Rolle zugewiesen als dies bei Schupperts Ansatz der Fall ist. Hatte dieser noch kurz davor das »Recht als Steuerungsinstrument « betrachtet, $7^{8}$ so soll jetzt die »Verwaltungsrechtswissenschaft als Steuerungswissenschaft « konzipiert werden, was nunmehr eine Neuausrichtung des Verwaltungsrechts dergestalt beinhaltet, dass dieses angesichts

\footnotetext{
$70 \mathrm{Als}$ ein Beispiel für eindeutig definierte Funktionen sei auf Mayntz, Soziologie der öffentlichen Verwaltung, I978, S. 44 ff., verwiesen.

71 Eifert (Fn. 65), S. 103.

72 Die Überschrift wurde dem von Vesting gefertigten Tagungsbericht, DVBl I07 (1992), S. I34, entnommen.

73 Vesting (Fn. I0), S. I 35 .

$74 \mathrm{Zu}$ der von Schmidt-Aßmann vertretenen Systemvorstellung vgl. Bumke, Relative Rechtswidrigkeit. Systembildung und Binnendifferenzierung im Öffentlichen Recht, 2004, S. 3 I ff.

75 Vesting (Fn. I0), S. I35.

76 Schmidt-Aßmann (Fn. 45), S. I7.

77 Vesting (Fn. I0), S. I35.

78 Schuppert (Fn. I4), S. 73 ff. Siehe auch Schuppert, Zur Neubelebung der Staatsdiskussion: Entzauberung des Staates oder »Bringing the state back in«?, Der Staat 28 (I989), S. 9r ff.
} 
der zu lösenden gesellschaftlichen Probleme bei beobachtbarem »Formwandel [der] politische(n) Aufgabenerfüllung «79 dennoch in die Lage ist, seinerseits das Verwaltungshandeln so zu steuern, dass dadurch individuelle und/oder kollektive Akteure gezielt beeinflusst werden bzw. gesellschaftliche Zustände verändert werden können.

Bei der Neuausrichtung des Verwaltungsrechts im Lichte der Steuerungsidee vermochte ein von R. Mayntz 1987 im Jabrbuch zur Staats- und Verwaltungswissenschaft veröffentlichter Beitrag mit dem Titel »Politische Steuerung und gesellschaftliche Steuerungsprobleme - Anmerkungen zu einem theoretischen Paradigma « ${ }^{80}$ insofern einen Grundkonsens zu stiften, als sich führende Reformjuristen immer wieder auf diesen Text beriefen. ${ }^{8 \mathrm{I}}$ So liegt es nahe, das von R. Mayntz im Jahrbuch 1987 präsentierte Steuerungsmodell kurz vorzustellen. Angeknüpft wird an die von Mayntz vorgegebene "alltagssprachliche Verwendung des Steuerungsbegriffs", wobei mit dem Hinweis, man steuere ein Auto, ein Schiff, deutlich wird, dass dieser Begriff vornehmlich »in technischen Zusammenhängen « gebraucht wird. ${ }^{82}$ Ferner wird im Jahrbuch-Text darauf aufmerksam gemacht, dass Steuerung »nicht nur gezielte Beeinflussung « heißt, sondern auch bedeutet, »ein System von einem Ort oder Zustand zu einem bestimmten anderen zu bringen.« Es wird also eine »aktorbezogene(.) Perspektive« eingenommen, wobei Steuerungshandeln und Steuerungswirkung auseinander zu halten sind. Das Steuerungshandeln ist also einem Steuerungssubjekt [dem Staat oder »handlungsfähige(n) soziale(n) Kollektive(n)«] zuzurechnen, das gezielt auf ein autonomes Steuerungsobjekt einwirkt, um dieses zu veranlassen, sich auf ein Steuerungsziel hin zu »bewegen «. Dies soll mit Hilfe verschiedener Steuerungsinstrumente bzw. -modi erreicht werden, die das Steuerungssubjekt gezielt einzeln oder kombiniert einsetzen kann: In der Form »regulierende(r) Steuerung (durch Gebote und Verbote) «, »finanzielle(r) Steuerung (durch Anreizsysteme und Subventionen), « oder »prozessuale(r) Steuerung (durch Verhandlungssysteme« bzw. Verfahren). ${ }^{8} 3$ Erforderlich sei außerdem ein Steuerungswissen hinsichtlich der »Wirkungsbeziehungen zwischen Steuerungsaktivitäten und Steuerungsergebnissen, « um eine zweckrationale Wahl der Steuerungsinstrumente bzw. -maßnahmen vornehmen zu können. ${ }^{8}{ }^{4} \mathrm{In}$ der Festschrift für R. Mayntz wird dieses Steuerungskonzept wie folgt kommentiert:

»Im Ergebnis läuft dieser Definitionsversuch auf einen hierarchischen oder zumindest extrem asymmetrischen Steuerungsbegriff hinaus, der ein handlungsfähiges Steuerungssub-

79 Mayntz, Politische Steuerung: Aufstieg, Niedergang und Transformation einer Theorie, in: von Beyme/ Offe (Hrsg.), Politische Theorien in der Ära der Transformation, PVS-Sonderheft 26/1995, I 996, S. I 57 ff.

80 Mayntz (Fn. I7), S. 89 ff., S. 9I ff. Das Jahrbuch 1987 wurde von Schuppert (Fn. 78) besprochen, der u.a. darauf hinweist, dass dessen Herausgeber (Ellwein, Hesse, Mayntz, Scharpf) »durchweg renommierte Sozialwissenschaftler (sind), die beeindruckend und bis in die Juristenzunft hinein folgenreich über Staat und Verwaltung publiziert haben«(S. 92).

8I Statt vieler: Schuppert (Fn. I4), S. 68 ff.; äußert sich Schmidt-Aßmann (Fn. 36) im ersten Band der Schriften zur Reform des Verwaltungsrechts noch zurückhaltend zum Steuerungskonzept, so gibt er beim vierten Band dieser Reihe diese Zurückhaltung auf. Siehe Schmidt-Aßmann, Verwaltungsorganisationsrecht als Steuerungsressource, in: Schmidt-Aßmann/Hoffmann-Riem (Hrsg.), Verwaltungsorganisationsrecht als Steuerungsressource, I997, S. I4 ff. Dort findet sich auch auf S. I4, Fn. I6, der Hinweis auf den Mayntz-Text von 1987, außerdem wird auf den grundlegenden Sammelband hingewiesen, den König und Dose unter dem Titel »Instrumente und Formen staatlichen Handelns 1993 herausgegeben haben. In diesem Band wird das von Mayntz im Jahrbuch vorgeschlagene Steuerungsmodell dahingehend charakterisiert, es handle sich um eine »stringente(.), fast schon technokratische(.) Vorstellung von Steuerung « (König/Dose, ebd., S. 9).

82 Mayntz (Fn. I7), S. 93 .

83 Lange/Braun (Hrsg.), Politische Steuerung zwischen System und Akteur. Eine Einführung, 2000, S. 24. 84 Mayntz (Fn. I7), S. 94. 
jekt mit einer expliziten Steuerungsintention einem Steuerungsobjekt mit Systemeigenschaften gegenüberstellt, das zwar nicht selbst strategisch handeln, aber sich eigendynamisch verändern kann. Der Steuerungsbegriff ist also enger als der Begriff der Handlungskoordination (der auch marktförmige oder solidarische Koordinationsmechanismen einschließen würde), und er soll auch nicht auf Formen der adaptiven oder vereinbarten horizontalen Selbstkoordination zwischen gleichberechtigten Akteuren angewandt werden. Kollektive Selbststeuerung ist zwar nicht definitorisch ausgeschlossen, setzt aber die Existenz einer ausdifferenzierten Steuerungsinstanz im zu steuernden System voraus. « ${ }^{85}$

Mit der akteurzentrierten (handlungstheoretischen) Ausrichtung dieses Steuerungskonzepts verbindet sich eine Ablehnung der von Systemtheoretikern vertretenen Auffassung von dezentraler Steuerung sowie des Begriffs der Selbststeuerung (den R. Mayntz überhaupt durch den Begriff der Selbstorganisation ersetzt sehen möchte). Aus der Perspektive eines akteurzentrierten Ansatzes kann dezentrale Steuerung nur Steuerungsverzicht heißen wie die Selbststeuerung von Steuerungsobjekten von vornherein den »Willen« des Steuerungssubjekts außer acht lässt. Ebenso entschieden ist die Absage an konkurrierende Theorieangebote: »Die Theorie selbstreferentieller und autopoietischer Systeme (Luhmann ...), auf die die Advokaten >neuer Rechtsformen sich vorzugsweise stützen, führt hier kaum weiter; sie scheint eher zu einer falschen Diagnose der real existierenden Steuerbarkeitsprobleme zu verleiten. ${ }^{86}$

Unübersehbar ist, wie R. Mayntz selbst einräumt, die mit einer »bewußt (...) hoch selektive(n) Betrachtungsweise verbundene staatszentrierte Ausrichtung dieses Steuerungsmodells, ${ }^{87}$ das dieses neben anderen Merkmalen mit der klassischen Steuerungsmetapher (Steuermanns- und Schiffsmetapher) gemeinsam hat, was fürs erste die Anziehungskraft des Mayntz'schen Steuerungsmodells für Juristen plausibel macht:

»Der in der sozialwissenschaftlichen Steuerungsdebatte verwendete Ausdruck der (politischen) Steuerung »lässt sich etymologisch auf eine der ältesten und beständigsten Metaphern des okzidentalen Denkens zurückführen: die Metapher des Steuerns eines Schiffes auf hoher See. Von Steuerungskunst und -wissen des Steuermannes (kybernétes) einer griechischen Triere hingen Wohl oder Untergang des gesamten Schiffes samt Besatzung ab. Der herausgehobenen Funktion des Kybernétes korrespondierte seine erhöhte (...) Sitzposition auf der Brücke der Triere. Platon (Politeía 488a-489d; Politikós 302a) erkennt hierin das Leitbild für die politische Regierung eines Gemeinwesens: die Staatskunst (techne politiké) orientiert sich an der Kunst der Steuerung eines Schiffes (techne kybernétike), mit dem Staatsmann als Kybernétes. Dieser muss in der Sphäre des Politischen Kunstfertigkeit und überlegenes Wissen (epistéme) aufweisen, um das Ziel (telos) des Gemeinwesens (polis) - vorgestellt als einheitliches Handeln der Polis-Gemeinschaft zum Zwecke ihres Überlebens in einer unsicheren Umwelt - zu erreichen. $\ll^{88}$

Bei näherer Betrachtung ergeben sich - neben der bereits herausgestellten Staatszentriertheit - erstaunliche Parallelen zwischen der klassischen Steuerungsmetapher und dem von R. Mayntz offerierten Steuerungsmodell. So verweist die

85 Gerhardt/Derlien/Scharpf, Werkgeschichte Renate Mayntz, in: Gerhardt/Derlien/Scharpf (Hrsg.), Systemrationalität und Partialinteresse. Festschrift für Renate Mayntz, I994, S. 42. Vgl. Mayntz/Nedelmann, Eigendynamische soziale Prozesse. Anmerkungen zu einem analytischen Paradigma, Kölner Zeitschrift für Soziologie und Sozialpsychologie 39 (1987), S. 648 ff. Das Konzept der »Eigendynamik « resultiert aus der Auseinandersetzung mit Luhmanns Konzept der Selbstreferentialität, mit dem es einerseits »verwandt « ist, von dem es sich andererseits insofern unterscheidet, als nunmehr im Rahmen eines handlungstheoretischen Ansatzes auf ein prozesshaftes Geschehen abgestellt wird. Hierüber sollte jedoch nicht die Warnung von Beymes vergessen werden, »Eigendynamik heißt (...) nicht mehr als snicht von außen steuerbar«.« Vgl. von Beyme, Der Gesetzgeber. Der Bundestag als Entscheidungszentrum, I 997, S. 29.

86 Mayntz (Fn. I7), S. го . Angespielt wird auf die Arbeiten von Teubner und Willke.

87 Mayntz (Fn. 17), S. 93. Ferner Lange/Braun (Fn. 83), S. 25.

88 Lange/Braun (Fn. 83), S. I8, unter Bezugnahme auf Meichsner, Die Logik von Gemeinplätzen. Vorgeführt an Steuermannstopos und Schiffsmetapher, I983, S. 27 ff., S. 46-50. Siehe auch Lang, Zu einer kybernetischen Staatslehre. Eine Analyse des Staates auf der Grundlage des Regelkreismodells, I 970. 
klassische Steuerungsmetapher einerseits auf die »Orientierung [Ausrichtung] an einem Ziel, «89 andererseits »sieht sich das Schiff (als in sich festgefügte, Schutz und Geborgenheit gewährende Einheit) einer fremdartigen und eigengesetzlichen Umwelt gegenüber, gegen die es sich dauerhaft zu behaupten hat. «9० Die das Schiff umgebende Umwelt erweist sich somit als bedrohlich, so dass die Schiffsmetapher auch für die besondere Bedeutung von Krisensituationen steht, ${ }^{11}$ es sei in diesem Zusammenhang nur an die Schlagworte vom Staatsversagen « oder von der »Krise regulativer Politik « erinnert. Schließlich setzt die Kunst, ein Schiff durch eine widrige Umwelt zu steuern, wie die Kunst des Regierens Sachverstand und Steuerungswissen voraus, welches »nur « eine zur Steuerungswissenschaft umgerüstete Verwaltungsrechtswissenschaft zur Verfügung stellen kann. Und wenn R. Mayntz, ausgehend von der »alltagssprachlichen Verwendung des Steuerungsbegriffs «, mit dem Hinweis, man steuere ein Auto, ein Schiff, auf dessen Benutzung in technischen Zusammenhängen aufmerksam macht, dann zeigt sie nicht nur die konventionelle Verwendung der Steuerungsmetapher auf, sondern unterstreicht, wenn auch möglicherweise unbeabsichtigt, eine wichtige Annahme der später noch zu behandelnden kognitiven Metapherntheorie.

Allein infolge der Staatszentriertheit kann es eigentlich nicht überraschen, dass sich auch die Reformjuristen von dem Mayntz'schen Steuerungsmodell angesprochen fühlten, was reformkritischen Autoren, wie Ossenbühl,92 nicht entgangen ist, führt dieser doch, an Schuppert gewandt, in einem Diskussionsbeitrag auf einem in Göttingen veranstalteten Symposion aus:

»Sie haben nämlich die Anatomie [des Verwaltungsrechts] aus der Sicht des Normgebers betrachtet, des Staates, der etwas durchsetzen möchte, der etwas anstrebt, «93 um dann von Schuppert zu erfahren: »Meine Überlegungen gehen in der Tat von der Perspektive des Staates aus, und ich habe die Steuerungsthematik immer vom Steuerungsakteur her gedacht. (...). Denn auch wenn ich vom Steuerungsakteur her denke - also von der staatlichen Regelungsperspektive - muß ich darüber nachdenken, wie das Steuerungsinstrument beschaffen sein muß, damit es beim Steuerungsadressaten den gewünschten Steuerungseffekt auslöst. Dazu gehört etwa, daß die steuernde Instanz über die Wirkungsbedingungen einer effektiven Steuerung Bescheid wissen und sicherstellen muß, daß die Steuerungsimpulse den Steuerungsadressaten auch wirklich erreichen. «94

Dieses Eingeständnis Schupperts kommt nicht unerwartet, hatte er doch bereits in einem frühen Beitrag unter expliziter Bezugnahme auf das Mayntz'sche Steuerungsmodell erklärt: »Geht es um Steuerung durch Recht, so steht als Steuerungsakteur der Staat und als Steuerungsinstrument das Recht im Vorder-

89 Meichsner (Fn. 88), S. 24, die auf das "Ziel einer Schiffsreise«, auf das Einlaufen des Schiffes in den sicheren Hafen hinweist.

90 Meichsner (Fn. 88), S. I9, eigene Hervorhebung, um auf die Parallele zur »Eigendynamik « hinzuweisen. 9I Meichsner (Fn. 88), S. 2 I.

92 Siehe seinen Diskussionsbeitrag auf der Dresdner Staatsrechtslehrertagung, VVDStRL 56 (I997), S. 283 f.

93 Diskussion zum Vortrag von Schuppert, Das Gesetz als zentrales Steuerungsinstrument des Rechtsstaates, in: Schuppert (Hrsg.), Das Gesetz als zentrales Steuerungsinstrument des Rechtsstaates. Symposion anläßlich des 60. Geburtstages von Christian Starck, I998, S. I05 ff. (Vortrag), S. I 57 ff. (Diskussion), S. 165 .

94 Schuppert (Fn. 93), S. I68, Diskussion. Überhaupt wird dieser Gesichtspunkt, auf den auch Pitschas in seinem nachstehend zitierten Diskussionsbeitrag aufmerksam macht, in der ganzen Diskussion bis heute stark vernachlässigt, nicht zuletzt deshalb, weil die Reformjuristen von einem normativen Steuerungsbegriff ausgehen: »Denn es gehört zur Eigenart des öffentlichen Rechts als Steuerungsmedium, daß es stets unter gegebenen strukturellen Bedingungen bestimmte Steuerungswirkungen entfaltet und andere eben nicht. (...). Auch verwaltungsrechtliche Steuerung folgt insofern bestimmten (gesetzlichen) Zielen; sie gründet auf Voraussetzungen, die sie selbst nicht garantieren kann und sie unterliegt Rahmenbedingungen, die das Steuerungsergebnis von vornherein präjudizieren. Siehe Schuppert, ebd., S. I63, Diskussion. Grundlegend zu dieser Thematik: Dreier, Hierarchische Verwaltung im demokratischen Staat. Genese, aktuelle Bedeutung und funktionelle Grenzen eines Bauprinzips der Exekutive, I99I, S. I 59 ff. 

von Recht mit dem die Rechtsordnung produzierenden Staat als >Steuermann<.«95

Die staatszentrierte Betrachtungsweise ist vor allem ein hohes Gut der traditionellen Staatslehre, so dass diese sich durchaus mit dem Steuerungsmodell hätte anfreunden können, gäbe es nicht weitreichende Differenzen, die sich beispielsweise mit dem Hinweis auf eine »veränderte Architektur von Staatlichkeit « ${ }^{6}$ und gewandelte Handlungsformen des Staates ${ }^{97}$ umschreiben lassen. Tiefergehende Differenzen zwischen Repräsentanten der traditionellen Staatslehre und Reformjuristen (Verwaltungsrechtlern) erhellt die kritische Auseinandersetzung, die Schulze-Fielitz bei der ausführlichen Besprechung des »Handbuchs des Staatsrechts « vornimmt. Schulze-Fielitz registriert eine nicht zu übersehende »(Über-)Betonung des Staates, « die seiner Meinung nach nicht nur darin zum Ausdruck kommt, dass offensichtlich »die klassischen Elemente der herkömmlichen Staatlichkeit im Völkerrecht als zentrale und angemessene Ausgangspunkte für die Analyse des modernen Verfassungsstaates « herangezogen werden, ${ }^{98}$ sondern auch darin eine Bestätigung findet, dass »eine Emanzipation vom Text des GG zugunsten einer Position [stattfindet], für die der Staat und seine Aufgaben als solche zu zentralen Bezugspunkten werden. «Dies wäre nicht zu beanstanden, wenn dies nicht bedeuten würde, dass »Staat und Staatlichkeit (...) Voraussetzungen der Verfassung und dieser logisch vorgeordnet « sind, d.h. die $»$ Verfassung (...) vom Staat her interpretiert (wird). $\ll 99$

95 Schuppert (Fn. I4), S. 73. Ähnlich schon Schuppert ([Fn. 78], S. 96) bei der Besprechung des ersten Bandes des Jahrbuchs zur Staats- und Verwaltungswissenschaft 1987, in dem Mayntz ihr Steuerungsmodell vorstellt: »Zur Steuerung gehört - dies ist seit der auf den Weggang Bismarcks aus der Regierungsverantwortung gemünzten Karikatur >Der Lotse geht von Bord ‘ Allgemeingut - ein fähiger Steuermann; Steuerung wird also schwierig, fehlt es an einem Steuerungszentrum."

In dem zuerst genannten Beitrag Schupperts ([Fn. I4], S. 8I) findet sich unter Bezugnahme auf Mayntz (Fn. 83 $_{3}$ ) bereits die Formulierung »regulated self-regulation «, die als »sehr glückliche Kombination von Steuerung durch Recht und Selbststeuerung « bezeichnet wird und in der späteren Reformdiskussion, die dem »Gewährleistungsstaat « gilt, eine große Rolle spielen wird. Vgl. Hoffmann-Riem, Von der Erfüllungs- zur Gewährleistungsverantwortung - eine Chance für den überforderten Staat, in: HoffmannRiem, Modernisierung von Recht und Justiz, 200I, S. 28 ff., mit dem Abschnitt: »Kombination von Regulierung und Selbstregelung «. Die oben angesprochene »glückliche Kombination« zielt auf die »Unverzichtbarkeit des Staates als gemeinwohlverpflichtete Problemlösungsinstanz« (Schuppert [Fn. 78], S. I04), eine Thematik, die für den Gewährleistungsstaat eine besondere Herausforderung darstellt. Statt vieler Hofmann, Verfassungsrechtliche Annäherungen an den Begriff des Gemeinwohls, in: Münkler/ Fischer (Hrsg.), Gemeinwohl und Gemeinsinn im Recht. Konkretisierung und Realisierung öffentlicher Interessen, 2002, S. 36, mit der hintersinnigen Formulierung: »Die Verwaltungsrechtswissenschaft will nun >die Gemeinwohlfähigkeit komplexer Regelungsstrukturen von staatlichen und privaten Akteuren (Trute) erforschen. (...). Jedenfalls hat der Gemeinwohlbegriff auf diesem Felde seit einigen Jahren eine bemerkenswerte Konjunktur. Wahrscheinlich wird er im ausufernden Prozeß partikularistischer Privilegierungen administrativer Funktionen als eine Art semantische Bremse gebraucht. « Einen Überblick gibt Große-Kracht, Die überraschende Renaissance des Gemeinwohls. Strohfeuer oder Auftakt zu einer neuen Debatte um das politische Selbstverständnis moderner Gesellschaften?, Soziologische Revue 27 (2004), S. $297 \mathrm{ff}$.

96 Trute, Die Wissenschaft vom Verwaltungsrecht: Einige Leitmotive zum Werkstattgespräch >Die Wissenschaft vom Verwaltungsrecht, $<$ Die Verwaltung, Beiheft 2, I999, S. Iо. Aus politikwissenschaftlicher Perspektive: Grande, Die neue Architektur des Staates. Aufbau und Transformation nationalstaatlicher Handlungskapazität - untersucht am Beispiel der Forschungs- und Technologiepolitik, in: Czada/ Schmidt (Hrsg.), Verhandlungsdemokratie, Interessenvermittlung, Regierbarkeit. Festschrift für Gerhard Lehmbruch, i 993 , S. 5 i ff.

97 Vgl. Schulze-Fielitz (Fn. 9), S. 274 ff. Siehe bereits Schulze-Fielitz, Der Leviathan auf dem Weg zum nützlichen Haustier?, in: Voigt (Hrsg.), Abschied vom Staat - Rückkehr zum Staat?, I993, S. 96 ff. Allerdings gesteht Schulze-Fielitz ([Fn. 9], S. 274) Kirchhof zu, er registriere und systematisiere in seinem Handbuch-Beitrag ( $\mathbb{S} 59$ ) »eindrucksvoll die Herausforderungen für die Verfaßtheit des Staates in den gewandelten Mitteln seines Handelns (...) in Entsprechung zum Wachstum und Wandel seiner Aufgaben (...) und im Bezugsrahmen des doppelten Dreiklangs von Rechtsetzen, Personaleinsatz und Finanzierung als >Blankettinstrumenten< (...) und von Aufgaben, Kompetenzen und Befugnisssen (...).«

98 Schulze-Fielitz (Fn. 9), S. 252. 
Dieser »Reanimierung klassischer Staatlichkeit « wird bescheinigt, sie entspreche möglicherweise nicht »der realen Bedeutung und Entwicklung moderner Staatlichkeit « und wirke »so empirisch und theoretisch anachronistisch.«Vor allem verkenne sie, dass es sich hierbei » weniger um einen Rückzug als um einen Funktionswandel moderner Staatlichkeit « handle, der - und hierauf kommt es besonders an - »in der Staatsrechtslehre der Gegenwart viel genauer, problemnäher und innovativer auf der Ebene des Verwaltungsrechts « erörtert werde. ${ }^{100}$ Lässt diese Formulierung noch offen, von wem diese verwaltungsrechtliche Diskussion bestritten wird, so benennt die dazugehörende Fußnote durch die Aufzählung der von Hoffmann-Riem und Schmidt-Aßmann (bzw. Schuppert) herausgegebenen Tagungsbände zur Reform des Verwaltungsrechts die führenden Repräsentanten der Gegenposition. Erfährt man zudem, dass beim »Handbuch des Staatsrechts « Autoren mit »klangvoll-renommierte(n) Namen aus der >mittelalterlichen< und der älteren Generation der deutschen Staatsrechtslehrer « vertreten sind, wie umgekehrt solche Autoren nicht zu finden sind, die »auf den Jahrestagungen (der Staatsrechtslehrervereinigung) der letzten zwei Jahrzehnte vor I 995 "als Vortragende aufgetreten sind (wie Hoffmann-Riem, Lange, Schuppert), ${ }^{10 I}$ dann dürfte die These nicht abwegig sein, bei dem von Hoffmann-Riem, Schmidt-Aßmann und Voßkuhle herausgegebenen, dreibändigen Handbuch zu den »Grundlagen des Verwaltungsrechts «, das den bisherigen Schlusspunkt der Reformdiskussion bildet, handelt es sich um ein ebenbürtiges Konkurrenzunternehmen zum »Handbuch des Staatsrechts«.

Trifft dies zu, dann stellt sich die (berechtigte) Forderung nach einer »Rückbindung der neuen Begrifflichkeiten [welche die Reformdebatte zum Verwaltungsrecht hervorgebracht hat] an die Dogmatik « nicht so zwingend. Insoweit wären auch die Tagungsbände wegweisende Vorarbeiten zum Handbuch des Verwaltungsrechts wie es auch im Rahmen einer solchen Publikationsform eher möglich ist, dass die Steuerungs-Perspektive durch die Governance-Perspektive ersetzt ${ }^{102}$ und dadurch eine gewisse Loslösung vom (ursprünglich) »staatszentrierten « Steuerungsparadigma vollzogen wird - unter Hinweis darauf, dass die schon beim kooperativen Staat erschwerte Unterscheidbarkeit von Steuerungssubjekt und Steuerungsobjekt sich völlig aufzulösen drohe, wenn, wie vom Gewährleistungsstaat gewollt, staatliche und nicht-staatliche Akteure zusammen Regelwerke (Normsetzung) erstellen und auch deren Vollzug gemeinsam implementieren. ${ }^{103}$

99 Schulze-Fielitz (Fn. 9), S. 248, S. 250; Meder, Ius non scriptum - Traditionen privater Rechtsetzung, Kap. 4, im Druck.

Io० Schulze-Fielitz (Fn. 9), S. 252 f.

IOI Schulze-Fielitz (Fn. 9), S. 245 f.

I02 Dies kündigt sich beispielsweise an mit dem von Trute/Denkhaus/Kühlers vorgelegten Beitrag »Governance in der Verwaltungsrechtswissenschaft«, DV 37 (2004), S. 45 I ff. Zuletzt Schuppert, $\mathbb{S} 6$ Verwaltungsorganisation und Verwaltungsorganisationsrecht als Steuerungsfaktoren, in: Hoffmann-Riem/ Schmidt-Aßmann/Voßkuhle (Hrsg.), Grundlagen des Verwaltungsrechts. Bd. I, 2006, S. I006 ff. Gegen den Governance-Begriff und für die Beibehaltung des Steuerungsansatzes spricht sich dagegen Voßkuhle (Fn. 8), S. 23 aus.

I03 Schuppert (Fn. I02), S. I006 f., mit Hinweis auf R. Mayntz, Governance Theory als fortentwickelte Steuerungstheorie?, in: Schuppert (Hrsg.), Governance-Forschung - Vergewisserung über Stand und Entwicklungslinien, 2005, S. I I. Vgl. Schuppert, ebd., S. I006: »In der sozialwissenschaftlichen Diskussion ist der Steuerungsbegriff in ebenso eindeutiger wie unumkehrbarer Weise durch den Begriff von Governance ersetzt worden. « Allein diese Hinweise dürften deutlich machen, dass die »vorausgesetzte Bedeutungsstabilität in Rezeptionsprozessen eher die Ausnahme bildet, so daß es unklar ist, worin dann noch die präskriptivierende und determinierende Kraft von Paradigmen besteht. Vgl. Danneberg, Sinn und Unsinn einer Metapherngeschichte, in: Bödeker (Hrsg.), Begriffsgeschichte, Diskursgeschichte, Metapherngeschichte, 2002, S. 399. 